\title{
chemosensors
}

ISSN 2227-9040

www.mdpi.com/journal/chemosensors

Review

\section{Simulation of Stimuli-Responsive Polymer Networks}

\section{Thomas Gruhn * and Heike Emmerich}

Material and Process Simulation (MPS), University of Bayreuth, Universitätsstr. 30,

D-95447 Bayreuth, Germany; E-Mail: heike.emmerich@uni-bayreuth.de

* Author to whom correspondence should be addressed; E-Mail: Thomas.gruhn@uni-bayreuth.de;

Tel.: +49-921-55-7873; Fax: +49-921-55-7899.

Received: 11 July 2013; in revised form: 19 September 2013 / Accepted: 4 November 2013 /

Published: 20 November 2013

Abstract: The structure and material properties of polymer networks can depend sensitively on changes in the environment. There is a great deal of progress in the development of stimuli-responsive hydrogels for applications like sensors, self-repairing materials or actuators. Biocompatible, smart hydrogels can be used for applications, such as controlled drug delivery and release, or for artificial muscles. Numerical studies have been performed on different length scales and levels of details. Macroscopic theories that describe the network systems with the help of continuous fields are suited to study effects like the stimuli-induced deformation of hydrogels on large scales. In this article, we discuss various macroscopic approaches and describe, in more detail, our phase field model, which allows the calculation of the hydrogel dynamics with the help of a free energy that considers physical and chemical impacts. On a mesoscopic level, polymer systems can be modeled with the help of the self-consistent field theory, which includes the interactions, connectivity, and the entropy of the polymer chains, and does not depend on constitutive equations. We present our recent extension of the method that allows the study of the formation of nano domains in reversibly crosslinked block copolymer networks. Molecular simulations of polymer networks allow the investigation of the behavior of specific systems on a microscopic scale. As an example for microscopic modeling of stimuli sensitive polymer networks, we present our Monte Carlo simulations of a filament network system with crosslinkers.

Keywords: hydrogel; polymer network; simulation; phase field theory; self-consistent field theory 


\section{Introduction}

If you put a gummy bear into a glass of water, the next day you will find the same bear grown in volume by more than a factor of five. (The effect depends on the choice of Gummy bears.) This is a very simple example of a polymer network that is strongly responsive to the stimuli of the environment (in this case humidity). Different kinds of smart polymer networks are sensitive to different stimuli, such as temperature changes [1,2], illumination [3,4], or properties of the solvent, such as the $\mathrm{pH}$ value [1,5-7], the ion concentration $[7,8]$, or the chemical composition [5,8-10]. Some materials are responsive to more than one kind of stimuli $[11,12]$. Reversible networks can be switched back and forth between different states by turning the stimulus on and off. Smart polymer networks can be used for various applications, such as chemical sensors [8,13], biosensors [13,14], actuators [7,15], artificial muscles [5], or for drug transport and release $[1,6,16]$. For the development and optimization of these devices, it is crucial to investigate the network behavior, both experimentally and theoretically. In this article, we focus on numerical methods for studying intelligent polymer networks and describe modeling techniques on different scales, ranging from macroscopic methods to molecular modeling.

There are two important types of smart polymer networks: (a) Covalently crosslinked networks that swell or shrink if exposed to a stimulus, and (b) networks with a variable topology, in which crosslinks can be broken or created by external stimuli.

Both types of smart polymer networks can change their density, their mechanical properties, and their permeability in response to changes of the environment. The majority of applications use chemically crosslinked polymer networks (see the application references in the first paragraph), however, networks with stimuli-sensitive crosslinks are also used for the development of sensors [9] and for controlled drug delivery and release $[5,17,18]$. One important aspect of reversibly crosslinked polymer networks is their ability to rebuild broken bonds and to rearrange in a completely different topology and shape. This way, the polymer network can heal if it has been plastically deformed or broken [19]. On the other hand, a fixed network topology has its own advantages: Even strongly swollen, the gummy bear still keeps its shape.

This article is dedicated to numerical studies of smart polymer networks that are sensitive to external stimuli. Modeling methods are discussed on different levels of details, from macroscopic theories to molecular modeling. The introduction to the different techniques is combined with more detailed descriptions of three numerical methods that have been developed by the authors. We think that they are particularly interesting examples of numerical studies of intelligent polymer networks that show novel routes for polymer network modeling [20-25]. We use these examples to describe important aspects of modeling methods on different levels of detail. For a comprehensive description of our numerical studies, we refer to the original articles [20-25].

We start with macroscopic models, in which all material properties are described by continuous fields. Here, we discuss various modeling principles, before we explain, in more detail, a phase field model for chemo-sensitive hydrogels that was recently presented by one of the authors [20]. Afterwards, we give a short introduction to self-consistent field theory. The starting point of the self-consistent field theory is the exact statistically physical description of a polymer model system so that constitutive equations are not required. It has been used, for many decades, for the studying of polymer melts. We elucidate an extension of the method that allows modeling reversibly crosslinked polymer 
networks with switchable microphases [21-23]. Finally, we discuss molecular modeling techniques and present, as an example, a Monte Carlo study of a network formed by stiff polymers and physical crosslinkers that, depending on external parameters, can change its structural and topological properties [24,25].

\section{Macroscopic Models, Finite Element and Phase Field Methods}

Stimulated by external conditions, such as a change of the $\mathrm{pH}$ or the chemical composition of the solvent, the volume of hydrogels can change strongly. For the investigation of hydrogel swelling on a macroscopic scale, various macroscopic models have been developed. Overviews on macroscopic hydrogel models have been presented in [26-28].

The first model, developed by Flory and Rehner [29,30], is based on the entropy changes in a swelling polymer network. There are various extensions of this statistical approach [31-34]. The equilibrium state of a hydrogel in a given environment can be calculated by minimizing the free energy of the total system. Relevant contributions to the free energy are the interaction and the entropy of polymer chains, the entropy of ions in the hydrogel and in the bath, and the Coulomb interactions. Thermodynamic hydrogel models have been used, for example, by Caykara et al. [35], and Kramarenko et al. [36].

The equilibrium ion distribution in the hydrogel and in the bath can be obtained from the Donnan equilibrium and the postulation of electric neutrality, as discussed below.

An important model for hydrogels is the multiphase mixture model, in which the system consists of two or more phases. In the biphasic theory, the system is divided into an incompressible polymer phase and an incompressible fluid phase that is in contact with a fluid reservoir [37]. The method has been extended to three-phase (or multiphase) mixture models that include a polymer phase, a solvent phase, and one or several ionic phases, formed by the mobile ions [38-41]. A multiphase approach, based on the theory of porous media, has been developed by Freiboth et al. [42].

Macroscopic hydrogel theories are frequently based on conservation laws and constitutive equations $[39,40,41,43]$. If the ion, solvent, and polymer phases are incompressible, mass conservation leads to:

$$
\phi_{p}+\phi_{s}+\sum_{k=1}^{N} \phi_{k}=1
$$

where $\phi_{p}$ and $\phi_{s}$ are the volume fractions of the polymer network and the solvent phase, while $\phi_{1}, \ldots, \phi_{N}$ are the volume fractions of the $N$ ion phases. The volume fractions $\phi_{k}$ are proportional to corresponding concentrations $c_{k}$. In the absence of chemical reactions, the concentrations obey the conservation law:

$$
\frac{\partial}{\partial t} c_{k}=\nabla_{\mu}\left(c_{k} v_{\mu}^{(k)}\right) \quad(k=p, s, 1, \ldots, N)
$$

where $v_{\mu}^{(x)}$ is the $\mu$ th component of the local velocity of phase $k$. In this article, we use the summation convention for Greek indices that appear twice.

Frequently, electric neutrality is postulated for the hydrogel system [41,43-45]. If negatively charged ions are attached to the polymer network with a concentration $c_{f}$, electric neutrality requires: 


$$
z_{f} c_{f}+\sum_{k=1}^{N} z_{k} c_{k}=0
$$

Here, $z_{f}$ and $c_{f}$ are the valence number and the concentration of fixed ions, attached to the polymer network and $z_{k}$ is the valence number of the mobile ion species $k$. In several models, the dynamics of the system is studied with the help of momentum equations [37-41,43,46]. For the solvent and the ion phases, the forces induced by the chemical potential of solvent molecules, and the electrochemical potentials of the ions, are balanced by the friction forces between the different phases that flow with different velocities. The equations are of the form:

$$
\rho_{k} \nabla_{\nu} \mu^{(k)}-\sum_{m \neq k} \alpha_{m, k}\left(v_{\nu}^{(m)}-v_{\nu}^{(k)}\right)=0 \quad(k=s \text { or } k=1, \ldots, N)
$$

with chemical/electrochemical potentials $\mu^{(k)}$. The mass densities $\rho_{k}$ are given by $\rho_{k}=m_{k} c_{k}$, where $m_{k}$ is the mass of a solvent molecule $(k=s)$ or an ion $(k=1, \ldots, N)$. The constants $\alpha_{m, k}$ are friction coefficients between phases $k$ and $m$. For the polymer phase, one has:

$$
\nabla_{\kappa} \sigma_{\kappa \lambda}=0
$$

where $\sigma_{\kappa \lambda}$ are the components of the stress tensor. Furthermore, several constitutive equations are used: Usually, the chemical potential is expressed by a linear combination of the ion concentrations and the hydrostatic pressure, while the electrochemical potentials of the ions can be expressed as a function of the chemical activity term, which is proportional to $\ln \left(\gamma_{k} c_{k}\right)$ with an activity coefficient $\gamma_{k}$, and a term that is proportional to the electric potential [39]. Furthermore, a linear or nonlinear relation between the stress tensor of the polymer network and its elastic strain tensor is considered [28].

If the ion concentrations in the solvent are small, so that $\phi_{s}$ and $\phi_{p}$ are distinctly larger than the volume fractions of the mobile ions $\phi_{k}(k=1, \ldots, N)$, Equation (1) can be approximated by $\phi_{P}+\phi_{S}=1$. Then, the volume fraction of water molecules is determined by the polymer volume fraction and does not have to be considered explicitly.

The flow of ions can be treated more generally by using the Poisson equation instead of the electric neutrality condition. Models that include the Poisson equation have been used, for example, by Wallmersperger et al. [47], Li et al. [48], and Chen et al. [39], to study the swelling and shrinking of hydrogels induced by chemical and electric stimuli.

Following the method used in [48], here we discuss a typical set of equations used in combination with the Poisson equation. The time dependence of the concentration $c_{k}$ of mobile ions of type $k$ can be described by the Nernst-Planck equation:

$$
\frac{\partial c_{k}}{\partial t}=-\nabla_{\mu} \cdot\left[D_{k}\left(\nabla_{\mu} c_{k}+\frac{z_{k} F}{R T} c_{k} \nabla_{\mu} \psi\right)\right], \quad(k=1, \ldots, N)
$$

with the Faraday constant $F \simeq 9.649 \times 10^{4} \mathrm{Cmol}^{-1}$, the gas constant $R \simeq 8.314 \mathrm{Jmol}^{-1} \mathrm{~K}^{-1}$, and the temperature $T$. Ions of species $k$ have diffusion constants $D_{k}$. The Nernst-Planck equation corresponds to the continuity equation Equation (2) under the assumption that the ion flow is determined by the concentration gradient and the electric potential $\psi$. For systems that show chemical reactions or a non-ideal mixing behavior of the solvent, extended versions of Equation (5) have been used, which include a term for the chemical activity or other source terms for the ions [49]. In [39], a convection 
term $-\nabla_{\mu}\left(c_{k} V_{\mu}\right)$, with a flow velocity $\underline{V}$, is added to the right side of Equation (5). The electric potential $\psi$ obeys the Poisson equation:

$$
\nabla^{2} \psi=-\frac{F}{\epsilon \epsilon_{0}}\left(\sum_{k=1}^{N} z_{k} c_{k}+z_{f} c_{f}\right)
$$

with the vacuum dielectric constant $\epsilon_{0}$ and the relative dielectric constant $\epsilon$. The osmotic pressure depends on the differences $c_{k}-c_{k}^{(e x t)}$ between the ion concentrations $c_{k}$ in the hydrogel and the respective concentrations $c_{k}^{(e x t)}$ at the external boundary of the hydrogel:

$$
p^{(o s m)}(\underline{x})=R T \sum_{k=1}^{N_{k}}\left(c_{k}(\underline{x})-c_{k}^{(e x t)}\right)
$$

The osmotic pressure causes a deformation $\underline{u}(\underline{x})$ of the initial geometry of the hydrogel. Strong, non-linear deformations can be considered by finite strain theory $[45,46,48]$. If the deformations are small, they fulfill the linear equation:

$$
\nabla_{\mu} P_{\mu \nu}=\nabla_{\mu}\left(\lambda_{h} E_{\lambda \lambda} \delta_{\mu \nu}+2 \mu_{h} E_{\mu \nu}-p^{(o s m)} \delta_{\mu \nu}\right)=0
$$

where $P_{\mu \nu}$ and $\delta_{\mu \nu}$ are components of the full pressure tensor in the hydrogel region and the unit tensor, respectively. The coefficients $\lambda_{h}$ and $\mu_{h}$ are the first and second Lamé coefficients of the hydrogel. The Green-Lagrangian strain tensor $E_{\mu \nu}$ is given by:

$$
E_{\mu \nu}=\frac{1}{2}\left(\frac{\partial u_{\mu}}{\partial x_{\nu}}+\frac{\partial u_{\nu}}{\partial x_{\mu}}+\frac{\partial u_{\lambda}}{\partial x_{\mu}} \frac{\partial u_{\kappa}}{\partial x_{\nu}}\right)
$$

For small deformations, the third term in the brackets can be neglected. Solving Equations (6)-(9), numerically, requires appropriate boundary conditions. If the hydrogel is firmly attached to a substrate, the corresponding surface region is fixed. For the remaining surface region, $\Gamma_{W}$, one has:

$$
P_{\mu \nu} n_{\nu}=W_{\mu} \quad \text { at } \Gamma_{W}
$$

with a surface normal vector $\underline{n}$ pointing outward and a surface traction vector $\underline{W}$.

Various types of stimuli-induced swelling and deformation of hydrogels have been investigated with transport theories. A numerical study of temperature-dependent swelling is presented in [50]. For hydrogel spheres in water, the radial polymer density distribution has been calculated as a function of swelling time [38]. Cheng et al. have studied the curling effect of articular cartilage with non-uniform fixed charge density [46].

A system of particular interest, which is frequently investigated experimentally [51], consists of a hydrogel strip in water between two electrodes. The electric field from the electrodes is perpendicular to the strip area. The strip is fixated at one end, or in the middle. The diffusion of ions, induced by the electric field, leads to a bending of the non-uniformly swelling strip. The deformation of the strip, which can be used for sensors and actuators in biological and other aqueous environments, could be reproduced and analyzed in detail in various numerical studies [39,43,52].

Equations (6)-(9) are typically solved with finite element methods. Alternatively, meshless methods have been used [39,40,50,53-56], which are conceptually more complex but avoid the definition and adaptation of a mesh grid during the simulation. In finite element and meshless methods, boundary conditions have to be defined on the hydrogel surface, which typically moves at each time step so that 
the hydrogel surface has to be traced during the swelling process. Recently, we have developed a phase field model, which avoids the precise localization of the phase boundaries and can be easily coupled with other phase fields that may describe additional phases in the system.

\subsection{Phase Field Theory}

The phase field method (PF method in the following) is generally used to study multiphase systems with interfacial dynamics. It allows following the dynamics of the system interfaces inherently coupled to all relevant physical and chemical driving fields. In recent years, there has been a growing number of articles that apply the PF method on polymer systems, i.e., systems in which at least one phase is a polymer melt or a polymer solution (see, for example, [57-61]). A phase field model for a hydrogel that has been developed in our group [20] is elucidated in detail below. To that end, we first introduce the concept of the PF method and describe how it has been applied on polymer systems. Our motivation for the model's development was to introduce a new modeling concept that can be easily adapted to different network properties and external conditions.

The PF method, which is used to describe the non-equilibrium dynamics of phase boundaries, is based on a free energy functional $F$. If there is only one phase field variable $\Psi(\underline{r}, t)$, the free energy is of the form:

$$
F=\int d V\left(f(\Psi)+f_{i n h}(\nabla \Psi)\right)
$$

where $f$ is the free energy density of the bulk and usually a double well potential. The second part considers spatial inhomogeneities. A simple example of the free energy in Equation (11) is the standard Ginzburg-Landau free energy:

$$
F=\int d V\left(c_{1}\left(\Psi^{2}-\Psi_{0}^{2}\right)^{2}+c_{2}|\nabla \Psi|^{2}\right)
$$

with coefficients $c_{1}$ and $c_{2}$. An overview over phase field methods and their applications are given in [62]. Usually, one has an extended region in space in which the phase field variable is $\Psi \simeq 1$ and another region in which the phase is absent and $\Psi \simeq 0$. Both regions are separated by an interface region of finite width, in which the phase field decreases smoothly. If $\Psi$ is conserved, the dynamics of the phase field is described by a Cahn-Hilliard equation [63]:

$$
\frac{\partial \Psi}{\partial t}=-\nabla \cdot \underline{J}
$$

Here, the flux density is given by:

$$
J=-M \nabla \frac{\delta F}{\delta \Psi}=-M\left(\frac{\partial F}{\partial \Psi}-\nabla_{\mu} \frac{\partial F}{\partial\left(\nabla_{\mu} \Psi\right)}\right)
$$

with a variational derivative $\delta F / \delta \Psi$, and a mobility $M$, which, in general, may depend on $\Psi$. In a fluid polymer blend, $\Psi$ is often taken as the concentration of one component and the bulk free energy density $f(\underline{r})$ is the Margules activity from the Flory-Huggins theory [64,65]. In many cases, a Gaussian noise term $\zeta(\underline{r} ; t)$ is added to the Cahn-Hilliard equation so that the equation has a Langevin form:

$$
\frac{\partial \Psi}{\partial t}=-\nabla \cdot \underline{J}+\zeta=\nabla \cdot\left(M \nabla \frac{\delta F}{\delta \Psi}\right)+\zeta .
$$


Considering a flow velocity $\underline{\nu}$, Equation (15) can be extended to the convective Cahn-Hilliard equation with a noise term [66]:

$$
\frac{\partial \Psi}{\partial t}+\underline{\nu} \cdot \nabla \Psi=-\nabla \cdot \underline{J}+\zeta=\nabla \cdot\left(M \nabla \frac{\delta F}{\delta \Psi}\right)+\zeta
$$

The fluid dynamics can be described by a continuity equation and a (advanced) Navier-Stokes equation, which is given by:

$$
\rho\left(\frac{\partial}{\partial t}+\underline{\nu} \cdot \nabla\right) \underline{\nu}=-\nabla P+2 \nabla \cdot\left(\underline{\underline{\sigma}}_{v i s c}\right)+\underline{k}_{\text {surf }}+\underline{k}_{e x t}+\underline{k}_{\text {elast }}
$$

where $\rho$ is the mass density, $P$ is the hydrostatic pressure, $\underline{\sigma}_{v i s c}$ is the viscous stress tensor, and $\underline{k}_{\text {surf }}$ considers the surface tension from the interfaces [59]. The term $\underline{k}_{e x t}$ represents external forces, such as gravity, while $\underline{k}_{\text {elast }} \propto\left(R_{0}^{-2}\langle\underline{R} \underline{R}\rangle-\underline{\underline{\mathbf{1}}}\right)$ denotes the elastic response of polymers to a flow field, leading to viscoelastic behavior [67]. An alternative description of viscoelasticity is the Oldroyd-B model [68], which has been used, together with the PF method, to study the elongation and burst of viscoelastic droplets [69] and the coalescence of polymer drops and interfaces [61,70].

The connection of the Cahn-Hilliard and the Navier-Stokes equation is called "Model H" in the nomenclature of Hohenberg and Halperin [71]. In many cases, the $k_{\text {elast }}$ part is neglected so that the polymer phase is described as a Newtonian fluid. In principle, all terms on the right hand side (beside $P$ ) can be coupled to the phase field variable $\Psi$, as the involved material parameters may depend on the composition. The model $\mathrm{H}$ has been used to study spinodal decomposition with and without external velocity fields and Rayleigh-Taylor instabilities [57,59,72]. The described method with slightly different Cahn-Hilliard free energy has been used to study the shape of fluid films on a dewetting substrate [73].

The dynamics of non-conserved phase field variables $\xi(\underline{r}, t)$ is described by the Allen-Cahn equation [74]:

$$
\frac{\partial \xi}{\partial t}=-M_{\xi} \frac{\delta F}{\delta \xi}
$$

where $M_{\xi}$ is the mobility coefficient and $F$ consists again of a bulk free energy density and a gradient term. In various studies, the Allen-Cahn equation has been used to investigate the growth of polymer crystals $[60,75,76]$. In the following example, it is used to characterize the extension of the hydrogel [20].

\subsection{Phase Field Model of a Hydrogel}

Recently, we have developed a PF model for studying the swelling behavior of a hydrogel as the ion concentration in the surrounding is changed [20]. In the following, some details are described to demonstrate the concept. The system is sketched in Figure 1. The extension of the hydrogel is characterized by a phase field variable $\xi(\underline{r}, t)$. The dynamics of $\xi$ is controlled by Equation (18), with a free energy [20]

$$
F=\int d V h(\xi)\left(\epsilon_{e l}-\Delta p\right)+\frac{W_{p}}{V_{m}} \xi(1-\xi)^{2}+\frac{K_{\xi}}{2}(\nabla \xi)^{2}
$$


The second term is a double well potential as used in Landau theory. The second and third term provide an interface region of finite width. $W_{p}$ and $V_{m}$ are the potential height and the molar volume of the mixture, respectively. In alloy systems, the coefficients $W_{p} / V_{m}$ and $K_{\xi}$ can be extracted from the surface energy and the interface width. For the function $h(\xi) \equiv \xi^{2}(3-2 \xi)$ one has $h(0)=0$, $h(1)=1$, and $h^{\prime}(0)=h^{\prime}(1)=0$, so that the derivative of the first part of $F$ is only finite in the interface region. The term $\Delta p \equiv p^{(o s m)}-p^{(e q)}$ is the difference between the osmotic pressure and $p^{(e q)}$, where $p^{(e q)}$ is the osmotic pressure at the initial equilibrium configuration from which the displacement $\underline{u}$ is measured. The osmotic pressure $p^{(o s m)}$ is defined as [20]:

$$
p^{(\text {osm })}=R T\left[\frac{\int d V \xi\left(\sum_{k=1}^{N} c_{k}\right)}{\int d V \xi}-\frac{\int d V(1-\xi)\left(\sum_{k=1}^{N} c_{k}\right)}{\int d V(1-\xi)}\right]
$$

The elastic energy density:

$$
\epsilon_{e l}=\frac{\lambda_{h}-\lambda_{s}}{2}\left(E_{\lambda \lambda}\right)^{2}+\mu_{h} E_{\mu \nu} E_{\mu \nu}
$$

includes the difference of the first Lamé coefficients in the hydrogel region $\lambda_{h}$ and in the surrounding bath region $\lambda_{s}$.

Figure 1. Sketch of a hydrogel system with a polymer network, a surrounding bath, ions attached to the network, and mobile cations and anions. In the phase field method, all system components are represented by continuous field variables. The extension of the hydrogel is characterized by a phase field variable, which is $\xi=1$ inside the hydrogel region (light green area). At the interface to the bath, the phase field decreases smoothly to $\xi=0[20]$.

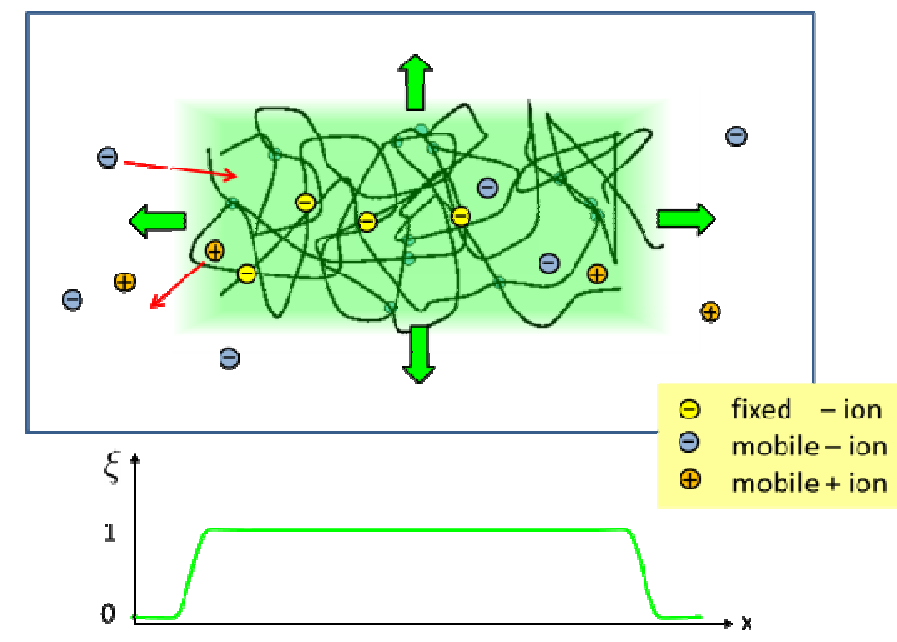

The bath solution is assumed to be a shear-free liquid. Analogously to Equation (8), we ensure mechanical equilibrium for the strain tensor $E_{\mu \nu}$ by an equation:

$$
\nabla_{\mu}\left(h(\xi) \sigma_{\mu \nu}^{(h)}+(1-h(\xi)) \sigma_{\mu \nu}^{(s)}-h(\xi) \Delta p \delta_{\mu \nu}\right)=0
$$

With $\sigma_{\mu \nu}^{(h)}=\lambda_{h} E_{\kappa \kappa} \delta_{\mu \nu}+2 \mu_{h} E_{\mu \nu}$ and $\sigma_{\mu \nu}^{(s)}=\lambda_{s} E_{\kappa \kappa} \delta_{\mu \nu}$. The concentration of fixed charges is defined by: 


$$
c_{f}(\underline{r}, t)=\frac{\xi(\underline{r}, t) c_{f}^{(e q)} \int d V^{\prime} \xi^{(e q)}\left(\underline{r}^{\prime}\right)}{\int d V \xi\left(\underline{r}^{\prime}, t\right)}
$$

where $c_{f}^{(e q)}$ and $\xi^{(e q)}$ are the concentration of fixed ions and the phase field variable at the initial equilibrium state. Note that $\int d V c_{f}(\underline{r}, t)=\int d V c_{f}^{(e q)}$ at all times. In the bath region, the concentration of fixed charges is zero inside the bath region while it is a finite constant inside the hydrogel region. The fields $\xi, c_{k}, c_{f}, \psi$, and $E_{\mu \nu}$ are coupled via Equations (19)-(23), which are solved simultaneously.

Figure 2. Profiles of the phase field $\xi(\underline{x})$ that represents the hydrogel region for boundary ion concentrations (a) $c_{b n d} \simeq 0,108 c^{*}$ and (b) $c_{b n d} \simeq 1.892 c^{*}$ [Springer, Colloid Polymer Science, 289, 2011, 513-521, Phase field model simulations of hydrogel dynamics under chemical stimulation, Li, D., Yang, H.L., Emmerich, H., Figure 4, (C) Springer-Verlag 2011] with kind permission from Springer Science+Business Media B.V.

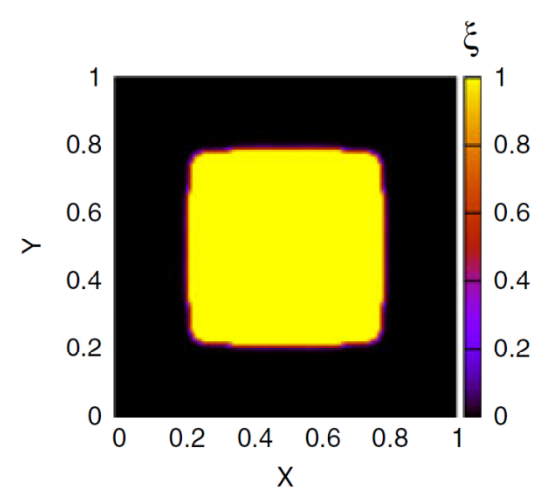

(a)

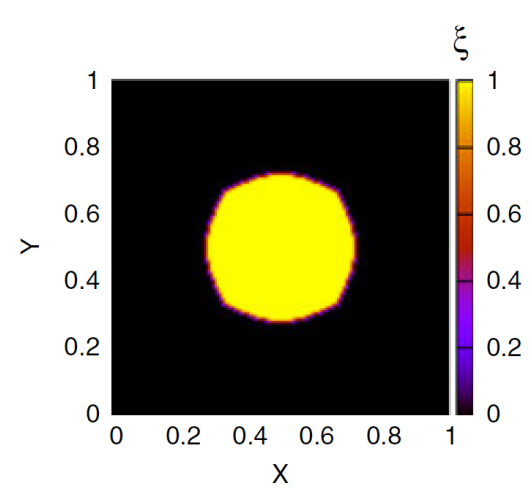

(b)

In the simulation, a hydrogel with negative fixed ions $\left(z_{f}=-1\right)$ is studied in a bath solution with $\mathrm{Na}^{+}\left(z_{1}=+1\right)$ and $\mathrm{Cl}^{-}\left(z_{2}=-1\right)$ ions. At the beginning, the concentration of fixed ions in the hydrogel is $c_{f}=4 \mathrm{~mol} \mathrm{~m} m^{-3}$. In the bath, the $\mathrm{Na}^{+}$and $\mathrm{Cl}^{-}$ions have a concentration of $c_{b}=2 \mathrm{~mol} \mathrm{~m}^{-3}$. The initial values of $c_{1}, c_{2}$, and $\psi$ in the hydrogel region are determined by the Donnan equilibrium and electric neutrality conditions

$$
c_{k}=c_{b} \exp \left(-z_{k} \Delta \Psi\right), \quad(k=1,2), \quad \sum_{k=1}^{2} z_{k} c_{k}+z_{f} c_{f}=0
$$

At the boundary of the system, the displacement and the electric potential is kept zero, while the concentration $c_{b n d}$ of mobile ions is continuously changed with time. The time step is chosen to be $0.05 s$.

In Figure 2, profiles of the phase field $\xi(\underline{x})$ are shown. At low ion concentration $c_{b n d} \simeq 0.108 c^{*}$ (Figure 2a) the gel is strongly swollen and shows a rather quadratic shape, due to the quadratic boundary of the simulation cell. At large ion concentration $c_{b n d} \simeq 1.892 c^{*}$ (Figure $2 \mathrm{~b}$ ) the hydrogel region becomes more circular in order to minimize the interface energy. 
Figure 3. Evolution of the gel fraction with time. In the shown time period, the boundary ion concentration is linearly decreased from $c_{b n d}=1 c^{*}$ to $c_{b n d} \simeq 0,108 c^{*}$, then increased to $c_{b n d} \simeq 1.892 c^{*}$, and decreased again to $c_{b n d}=1 c^{*}$ [Springer, Colloid Polymer Science, 289, 2011, 513-521, Phase field model simulations of hydrogel dynamics under chemical stimulation, Li, D., Yang, H.L., Emmerich, H., Figure 7, (C) Springer-Verlag 2011] with kind permission from Springer Science+Business Media B.V.

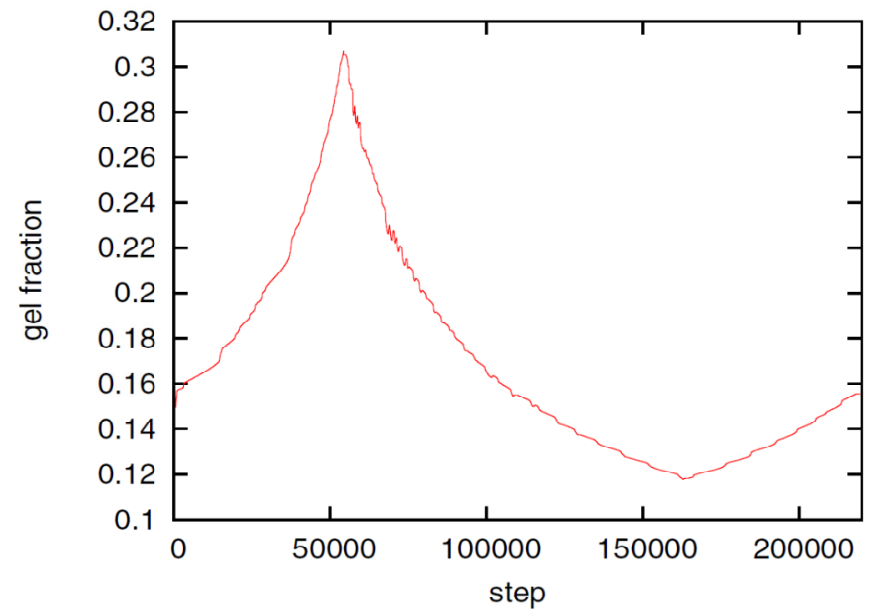

Figure 3 demonstrates the swelling, shrinking and swelling dynamics of the hydrogel as the boundary ion concentration is decreased to $c_{b n d} \simeq 0,108 c^{*}$ (time step 54,000), increased to $c_{b n d} \simeq 1.892 c^{*}$ (time step 163,500), and decreased again to the initial value. The gel fraction follows the changes of the boundary ion concentration with a short delay, which is too small to be visible in Figure 2 but has been investigated in the numerical study [20]. In general, the equilibrium gel volume is monotonously shrinking with increasing ion concentration in the bath. The result is in qualitative agreement with experiments, in which the $\mathrm{pH}$ is kept constant and the ion concentration is varied [35,77].

Our new phase field model is an alternative approach for studying the dynamics of hydrogel swelling induced by the ion concentration of the bath. With the chosen decrease or increase rate $\left|\dot{c}_{\text {bnd }}\right| \simeq 71 \mathrm{~mol} \mathrm{~m}^{-3} \mathrm{~s}^{-1}$ for the boundary ion concentration, the hydrogel swells and shrinks without significant hysteresis. The method goes without explicit boundary conditions at the hydrogel surface and can be easily adapted to other hydrogel systems. For example, one can study a hydrogel shell that includes an active agent. Then, a concentration field of drug molecules can be added to the given model in order to study the drug release kinetics.

\section{Self-Consistent Field Theory}

The macroscopic theories of polymer networks depend on phenomenological, constitutive equations. Microscopic aspects of the polymer system can be studied with molecular simulations but they are restricted to small time and length scales. An alternative method for polymer modeling is the self-consistent field theory. Here, polymers are represented by continuous lines. They interact by steric and attractive interactions, where the latter is weighted by the Flory parameter. The method takes into account the connectivity of polymer chains, the fact that two spatially separated monomers are much more correlated if they belong to the same polymer. For the given model system, the theory starts from 
an exact partition function. Then, the equilibrium properties are typically determined with the help of a saddle-point approximation. The self-consistent field theory, which has been introduced by Edwards and Helfand [78,79], is a well-established method for polymer chains without crosslinks [80-84]. Polymer networks with permanent random crosslinks have been studied with a replica field theory [85-87], but the application of the method in numerical studies is difficult, since the fields are defined in the replica space. Recently, we have developed an extended self-consistent field theory, which allows studying smart polymer networks with reversible crosslinks [21-23]. In Section 2.1, an application of the method for a blend of reversibly crosslinked $A B$ diblock copolymers, mixed with $A$ and $B$ homopolymers, is described. The monomers $A$ and $B$ are assumed to have a low compatibility so that the correspnding homopolymers would demix macroscopically in the absence of the $A B$ copolymers. In the $A B+A+B$ mixture, various nanostructures may form. Without reversible crosslinks, the system has been studied in detail theoretically [81,88,89] and experimentally [90,91]. Many aspects of the model can be transferred to other systems with amphiphilic $A B$ copolymers dissolved in a mixture of incompatible solvents. We assume that the $A B$ copolymers can bind with non-covalent crosslinks, as in [92].

The model system resembles an incompressible polymer blend with a constant overall density $\rho_{0}$ and three types of polymers $j=a, b, c$. The system consists of (i) $n_{a}$ homopolymers of type $a$ with $A$ monomers and a length $N_{a}$, (ii) $n_{b}$ homopolymers of type $b$ with $B$ monomers and a length $N_{b}$, (iii) $n_{c}$ polymers of type $c$, which are symmetric diblock copolymers $A B$ that have a length of $N_{c} \equiv N$. The system stoichiometry is determined by the volume fraction of copolymers:

$$
\phi_{c}=\frac{n_{c} N}{\sum_{j=a, b, c} n_{j} N_{j}}
$$

and the relative $A$ fraction of homopolymers:

$$
\phi_{a}^{r e l}=\frac{n_{a}}{n_{a}+n_{b}}
$$

We use paths $\underline{R}_{j, k}(s)$ with $s \in\left[0, N_{j}\right]$ to parametrize the configuration of the $k$ th polymer of type $j$. All chains have a Gaussian shape distribution $\mathcal{P}\left(\underline{R}_{j, k}(s)\right)$. $A$ and $B$ monomers have a local density $\hat{\rho}_{A}(\underline{x})$ that can be divided into the density $\hat{\rho}_{A}^{(0)}(\underline{x})$ from type $a$ homopolymers and the density $\hat{\rho}_{A}^{(1)}(\underline{x})$ from the $A$ part of the copolymers. Analogously, one has $\hat{\rho}_{B}(\underline{x})=\hat{\rho}_{B}^{(0)}(\underline{x})+\hat{\rho}_{B}^{(1)}(\underline{x})$ for the density of $B$ monomers.

Without crosslinks, the partition function of the system is given by:

$$
Z_{n b}=\prod_{j=a, b, c} \frac{1}{n_{j} !} \prod_{k=1}^{n_{j}} \int \hat{\mathcal{D}}\left\{\underline{R}_{j, k}\right\} \mathcal{P}\left(\underline{R}_{j, k}(s)\right) \eta_{n b}
$$

with path integrals $\int \hat{\mathcal{D}}\left\{\underline{R}_{j, k}\right\}$ over all configurations of the $k$ th polymer of type $j$. Path integrals are useful mathematical tools. They can be considered as the limit for $N \rightarrow \infty$ of a $3 N$-dimensional integral that considers all configurations of a polymer with fixed length $L$ and $N$ atoms of diameter $L / N$. In practice, most path integrals cannot be solved numerically. One exception is the path integral of a single Gaussian chain. The basic idea of the self-consistent field theory is to transform Equation (27) such that only path integrals of single Gaussian chains have to be solved. In Equation (27), non-bonded interactions between polymers are considered by: 


$$
\eta_{n b} \equiv \delta\left(\hat{\rho}_{A}+\hat{\rho}_{B}-\rho_{0}\right) \exp \left(-\frac{1}{\rho_{0}} \int \mathrm{d} x \chi \hat{\rho}_{A} \hat{\rho}_{B}\right)
$$

The Flory-Huggins interaction in the exponent includes a Flory parameter $\chi$. The delta function considers the incompressibility of the polymer blend. So far, the ansatz corresponds to that of Edwards and Helfand [78,79]. We have developed an extended model, which considers the formation of reversible crosslinks. The result is the first self-consistent field theory for reversibly crosslinked polymer networks. The method is described in detail in [21]. In the following, we explain the basic concept.

\section{Self-Consistent Field Theory for Reversibly Crosslinked Polymer Networks}

We apply our extended self-consistent field theory on an $A B+A+B$ polymer blend that may form reversible crosslinks between the copolymers. We assume that crosslinks between adjacent monomer pairs $A+A, A+B$, or $B+B$ form with a probability proportional to $z \omega_{A} \omega_{A}, z \omega_{A} \omega_{B}$, or $z \omega_{B} \omega_{B}$, respectively. Here, $z$ is the crosslink strength and $\omega_{A}$ and $\omega_{B}$ are weighting factors, considering the types of the crosslinked monomers. The partition function can then be written as [21-23]:

$$
Z=\prod_{j=a, b, c} \frac{1}{n_{j} !} \prod_{k=1}^{n_{j}} \int \hat{\mathcal{D}}\left\{R_{j}\right\} \mathcal{P}\left(\underline{R}_{j, k}(s)\right) \eta_{n b} \exp \left(z \int \mathrm{d} x\left(\omega_{A} \hat{\rho}_{A}^{(1)}+\omega_{B} \hat{\rho}_{B}^{(1)}\right)^{2}\right)
$$

Setting $\omega_{A}+\omega_{B}=1$, we use the crosslink strength $z$ and the crosslink asymmetry $\Delta \omega \equiv \omega_{A}-\omega_{B}$ to specify the crosslink properties. For convenience, the crosslink strength is represented by the parameter $z_{\text {rel }}=z \rho_{0} / \chi$.

With the help of the Hubbard-Stratonovich (HS) transformation a free energy can be derived, which can be solved with a saddle-point approximation. We present the resulting free energy expression with dimensionless quantities, indicated by a tilde. Dimensionless lengths are defined as $\tilde{x}=x / R_{g}$ with the gyration radius $R_{g}$ of a free Gaussian polymer of length $N$. Dimensionless densities are given by $\tilde{\rho}_{A}=\rho_{A} / \rho_{0}$, while we use dimensionless free energies of the form $\tilde{F}=F /\left(k_{B} T \rho_{0} R_{g}^{d} N^{-1}\right)$ for a system with $d$ dimensions. We use $z_{r e l}=z \rho_{0} / \chi$ for the crosslink strength. The dimensionless mean field free energy can be divided into three terms [21]:

$$
\tilde{F}_{t o t}=\tilde{F}_{\chi}+\tilde{F}_{\omega}+\tilde{F}_{\text {en }}
$$

with a Flory-Huggins part:

$$
\tilde{F}_{\chi}=\frac{1}{k T} \int d \tilde{x} \chi N \tilde{\rho}_{A} \tilde{\rho}_{B}
$$

a crosslink contribution:

$$
\tilde{F}_{\omega}=\frac{1}{k T} \int d \tilde{x} z_{r e l} \chi N\left(\omega_{A} \tilde{\rho}_{A}^{(1)}+\omega_{B} \tilde{\rho}_{B}^{(1)}\right)^{2}
$$

and a free energy term:

$$
\tilde{F}_{\text {en }}=-\frac{1}{k T} \int d \tilde{x}\left(\tilde{p}\left(\tilde{\rho}_{A}+\tilde{\rho}_{B}-1\right)+\sum_{m=A, B} \sum_{j=0,1} \tilde{w}_{m}^{(j)} \tilde{\rho}_{m}^{(j)}\right)-\frac{1}{k T} \sum_{k=a, b, c} \frac{\phi_{k}}{N_{k}} N \ln \frac{Q_{k}}{n_{k}}
$$

which considers the entropy of the incompressible polymer blend for given average monomer densities. The auxiliary fields $\tilde{p}(\underline{x})$ and $\tilde{w}_{X}^{(j)}(\underline{x})$ with $X=A, B$ and $j=0,1$ are introduced in the HS transformation. The quantity $Q_{k}$ is the partition function of a single polymer chain of type $k$ fluctuating in the auxiliary fields (see [20]). It can be calculated numerically by solving modified 
diffusion equations. In the saddle point approximation, the auxiliary fields $\tilde{w}_{X}^{(j)}(\underline{x})$ and $\tilde{p}(\underline{x})$ and, finally, the desired monomer densities $\tilde{\rho}_{X}^{(j)}(\underline{x})$ can be determined by considering that the variations of the free energy with respect to $\tilde{w}_{X}^{(j)}(\underline{x}), \tilde{\rho}_{X}^{(j)}(\underline{x})$, and $\tilde{p}(\underline{x})$ must vanish. An algorithm is described in [21].

The method has been used to calculate a phase diagram as a function of the relative adhesion strength $z_{\text {rel }}$, the crosslink asymmetry $\Delta \omega$, and the relative $A$ fraction of homopolymers The investigated system has homopolymers of lengths $N_{a}=N_{b}=N / 5$ with $N \equiv N_{c}$, a Flory parameter of $\chi N=11.3$ and a volume fraction $\phi_{c}=0.6$ of copolymers. In the studied parameter range, the system can form a lamellar phase or a hexagonal phase, as shown in the sketches and the calculated $A$ monomer densitities in Figure 4 . The phase diagram has been determined by studying unit cells with a box length ratio of $L_{y} / L_{x}=\sqrt{3}$. The geometry supports the formation of a hexagonal as well as a lamellar phase. We have varied $L_{x}$ in the range of $3.0 \leq L_{x} \leq 4.8$ and determined the phase with the lowest free energy density. We find that for $\phi_{a}^{r e l}<0.58$ and $z_{\text {rel }}>0$, only lamellar structures are stable. Otherwise, the hexagonal phase is stable if $z_{\text {rel }}$ and $|\Delta \omega|$ are suitably small. In Figure 5, the phase boundaries between the hexagonal and the lamellar phase are shown for various $A$ fractions of the homopolymers in the range of $0.5 \leq \phi_{A}^{r e l} \leq 0.75$.

The self-consistent field theory study shows that the reversibly crosslinked polymer network in an $A B+A+B$ blend switches from the hexagonal phase to the lamellar phase and back if the stoichiometry of the system or the crosslink properties are changed. The latter switching method can be achieved with crosslinks that depend on external parameters, such as the temperature [2] or illumination [3]. Note that in the hexagonal structure, the minority phase is separated into small domains, while in the lamellar phase the stripes are extended on a large length scale. This may be used for drug release applications.

Figure 4. Nanostructures formed by a polymer blend of A and B homopolymers and reversibly crosslinked AB block copolymers. Shown are sketches of (a) the hexagonal phase and (b) the lamellar phase. Blue and yellow lines represent the A and B parts of the copolymers, yellow circles denote crosslinkers. A and B homopolymer densities are indicated by the light blue and yellow background colors. Results of self-consistent field theory calculations for the monomer density $\rho_{A}(\underline{x})$ in $(\mathbf{c})$ a hexagonal, and (d) a lamellar structure [22]. Figure (a) and (b) reproduced with permission from Thomas Gruhn, Heike Emmerich: Phase behavior of polymer blends with reversible crosslinks-A self-consistent field theory study. Journal of Material Research; Materials Research Society, in preparation, doi:10.1557/jmr.2013.315.

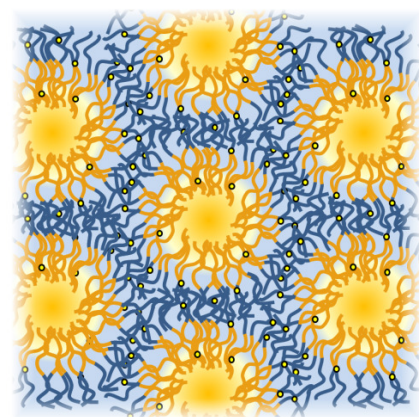

(a)

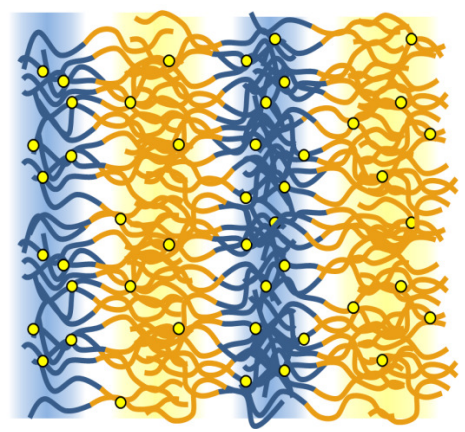

(b) 
Figure 4. Cont

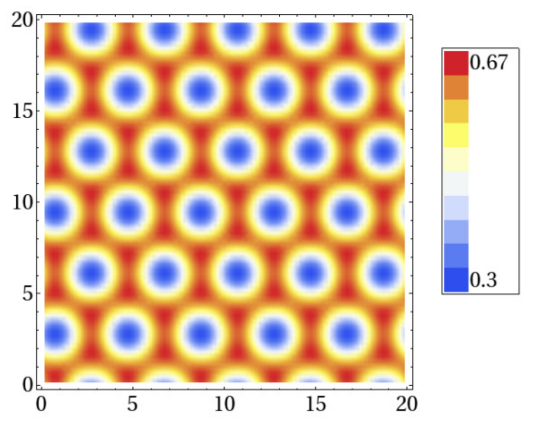

(c)

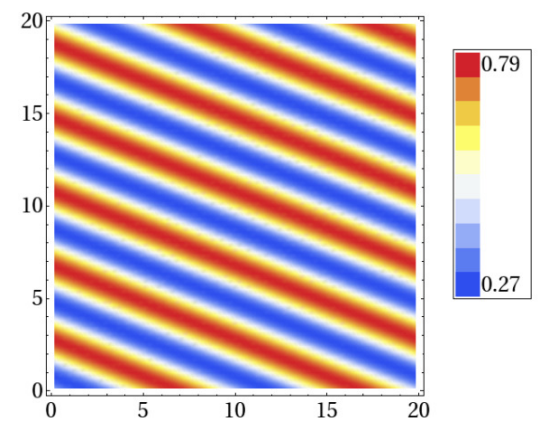

(d)

Figure 5. Phase diagram of the $A B+A+B$ polymer blend: The colored curves denote the phase boundaries between the hexagonal phase on the inside (small $\mid \Delta \omega)$ and the lamellar phase on the outside. Phase boundaries are shown for $\phi_{A}=0.75$ (dark blue), $\phi_{A}=0.7$ (red), $\phi_{A}=0.65$ (green), $\phi_{A}=0.6$ (brown), $\phi_{A}=0.59$ (magenta), $\phi_{A}=0.58$ (cyan) [22] Reproduced with permission from Thomas Gruhn, Heike Emmerich: Phase behavior of polymer blends with reversible crosslinks-A self-consistent field theory study, Journal of Material Research; Materials Research Society, in preparation, doi:10.1557/jmr.2013.315.

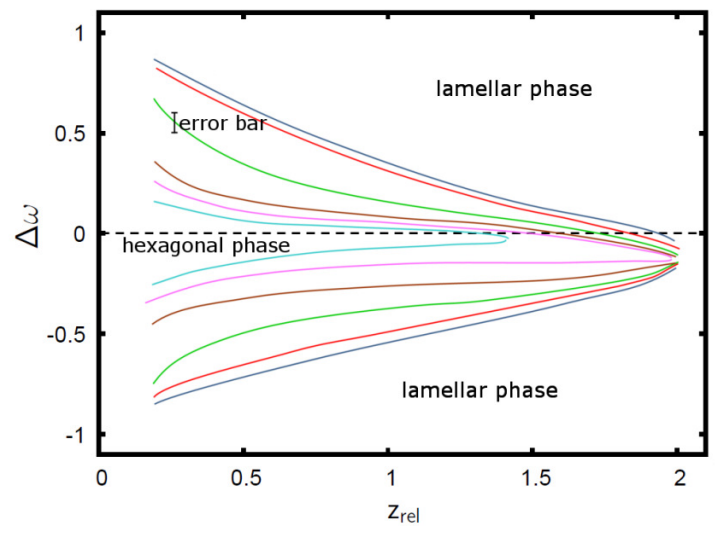

\section{Monte Carlo and Molecular Dynamic Simulations of Polymer Networks}

Properties of polymer networks can be studied on a microscopic level with the help of Monte Carlo simulations and molecular dynamic simulations, in which molecular details of the system can be investigated explicitly. Detailed descriptions of molecular simulation techniques can be found in [93] and [94]. Special simulation methods for polymer systems are described in [95]. In canonical Monte Carlo (MC) simulations, small changes of the molecule configurations are performed randomly at each step and the new configuration is accepted with a probability depending on the energy change. The acceptance criterion produces a sequence of configurations with a Boltzmann distribution. Other ensembles, such as a grand-canonical ensemble or a constant-pressure ensemble can be sampled by allowing changes of system parameters like the number of molecules or the volume of the system. In molecular dynamics (MD) simulations, the positions and velocities of the atoms are obtained from equations of motion that depend on external and interatomic forces. The method allows direct investigation of the dynamical properties of the system. Similar simulation techniques are Brownian dynamic simulations that include a random noise term and diffusive particle dynamic (DPD) 
simulations, in which the dynamics of interpenetrating spheres resemble the flow behavior of different species in the system (see $[93,94]$ ). DPD simulations have been used to investigate the release of colloids from stimuli-responsive microgel capsules [96]. Polyelectrolytes in electric fields have been studied with Brownian dynamics [97]. Compared to field theories, molecular simulations provide more details but are restricted to much smaller time and length scales. In multiscale simulations, material parameters obtained in molecular simulations are used in field-based or other coarse-grained methods on larger scales.

Atomistic simulations of polymers are very time consuming. Consequently, only a few atomistic simulations of polymer networks have been performed [98-101]. In a multiscale simulation of curing reactions in epoxy networks, atomistic simulations have been combined with DPD simulations [102]. The simulation effort can be reduced by using a coarse-grained model, such as the united atom model [103]. In this case, a polymer is approximated by a chain of beads, where each bead represents a monomer or a sequence of neighboring monomers. A model potential for a polymer chain is often composed of a bond length potential $u_{b l}$, which depends on the distance of neighboring beads, a bending potential $u_{\text {bend }}$, which considers the angle between neighboring bond vectors, and a potential $u_{\text {tors }}$ for the local torsion of the polymer chain. Then, the potential energy of a linear polymer with $N$ beads is given by:

$$
U_{\text {poly }}=\sum_{i=2}^{N} u_{b l}\left(\left\|\underline{r}_{i, i-1}\right\|\right)+\sum_{i=2}^{N-1} u_{\text {bend }}\left(\theta_{i}\right)+\sum_{i=2}^{N-2} u_{\text {tors }}\left(\phi_{i+1, i}\right)
$$

where $\underline{r}_{i, i-1}$ is the distance vector between the centers of mass of neighbor atoms $i-1$ and $i, \theta_{i}$ is the angle between $\underline{r}_{i+1, i}$ and $\underline{r}_{i, i-1}$, while $\phi_{i, i+1}$ is the angle between the vectors $\underline{r}_{i, i-1} \times \underline{r}_{i+1, i}$ and $\underline{r}_{i+1, i} \times \underline{r}_{i+2, i+1}$. This type of potentials has been used in Gibbs ensemble MD simulations of swelling polymer networks [104]. In a more coarse-grained approach, each bead can represent a chain sequence that is distinctly longer than the persistence length of the polymer. In this case, the angular dependent parts of the polymer potential can be neglected. The remaining bond length potential is typically modeled by a harmonic spring or a finitely extensible nonlinear elastic (FENE) potential [105-107]. The resulting chains are rather flexible and can be used to represent suitably long polymers on the respective length scale. If the persistence length is comparable with the polymer length, which is often the case for short biopolymers, a wormlike chain model is frequently used, which includes the bond-length and the bending potential but neglects the torsion [108,109]. In the case of very stiff polymers, one can also neglect the bending and bond-length fluctuations and represent the whole polymer by a rigid rod. While this approach neglects many internal degrees of freedom, it enables very efficient sampling of the configuration space. We elucidate the method and facilities of rigid rod simulations, using the example of our filament network simulations [24,25].

\section{Monte Carlo Simulation of Filament Networks with Reversibly Binding Crosslinkers}

We have used this method by studying a system of rigid filaments that can form filament networks with the help of reversibly binding crosslinkers [24]. One example for such systems are mixtures of short actin biopolymers and myosin crosslinkers. The system can form various structures, such as dissolved actin filaments, separated bundles of actin filaments, and filament networks with and without bundle structures. In living cells, the filament network is a dynamic system. Using ATP, the actin 
polymers can change their length and the myosin crosslinkers can perform directed walks along the actin filaments. In vitro, in the absence of ATP, the system is passivated and the actin polymers have a fixed length. Filaments with lengths in the range of the persistence length can be created with the help of suitable proteins. The resulting biomimetic hydrogels are of great interest as they are biocompatible and the structure of the network depends sensitively on the filament density, the crosslinker-filament ratio, and the temperature, and can be altered reversibly, by mechanical, chemical, or temperature stimuli.

We have studied the system with the help of Monte Carlo simulations. The filaments are represented by long hard spherocylinders, while the crosslinkers are modeled by short spherocylinders with specifically binding ends. A snapshot of the system is given in Figure 6a. The spherocylinders have a diameter $D$ and the cylindrical part has a length of $L=\ell_{F}$ and $L=\ell_{L}$ for filaments and crosslinkers, respectively. The configuration of a filament $j$ is determined by the center of mass $\underline{r}_{j}$ and a unit vector $\underline{u}_{j}$ that is parallel to the cylinder axis. Steric interactions between spherocylinders $i$ and $j$ are considered by a potential $U_{\text {steric }}\left(\underline{r}_{i}, \underline{u}_{i} ; \underline{r}_{j}, \underline{u}_{j}\right)$ that gets infinite if the two spherocylinders overlap. Each crosslinker, $i$, has adhesive sites at the both ends, with which it can physically bind to a neighboring filament, $j$. The definition of the adhesion potential is based on the shortest distances between the axis of filament, $j$, and the adhesive sites of the crosslinker, $i$ :

$$
\sigma_{i j}^{ \pm} \equiv \min _{-1 \leq s_{i} \leq 1}\left\|\left(\underline{q}_{j}^{ \pm}\right)-\left(\underline{r}_{i}+\frac{s_{i} \ell_{F}}{2} \underline{u}_{i}\right)\right\|
$$

with $\underline{q}_{j}^{ \pm} \equiv \ell_{a} \underline{u}_{j}$. The centers of the adhesive sites are localized on the crosslinker axis a distance $\ell_{a}$ away from the crosslinker's center of mass. We use $\ell_{C}=2 D$ and $\ell_{a}=1.35 D$, so that the adhesion sites lie inside the hemispheres of the crosslinkers.

The adhesion is mimicked by a square-well potential:

$$
U_{s w}\left(\sigma_{i j}^{ \pm}\right) \equiv\left\{\begin{array}{cl}
-\epsilon & \text { if } \sigma_{i j}^{ \pm}<a \text { and } \ell_{i} \neq \ell_{j} \\
0 & \text { else }
\end{array}\right.
$$

where $\epsilon$ is the potential depth. The condition $\ell(i) \neq \ell(j)$ denotes that crosslinkers can only adhere to filaments (which have a monodisperse length distribution). With a potential range $a=0.7 D$ the crosslinker can only bind to the filament if the surface of the spherocylinders are closer than $0.05 D$ and if the crosslinker axis is almost perpendicular to the filament surface. The total energy is given by:

$$
U=\sum_{i \neq j}\left(U_{\text {steric }}\left(\underline{r}_{i}, \underline{u}_{i}, \ell(i) ; \underline{r}_{j}, \underline{u}_{j}, \ell(j)\right)+U_{s w}\left(\sigma_{i j}^{+}\right)+U_{s w}\left(\sigma_{i j}^{-}\right)\right)
$$

The filament hydrogel is modeled with the help of a Metropolis algorithm. At each step, position and/or orientation of a filament or a crosslinker is changed randomly by a small amount, and the new configuration is accepted with a probability $P_{a c c}=\min \left(1, \exp \left(-\Delta U /\left(k_{B} T\right)\right)\right.$, where $\Delta U$ is the change of the configurational energy.

In the simulations, we have studied the occurrence of filament bundles and the percolation behavior of the system. A cluster is defined as a set of filaments that are interconnected by crosslinker bonds. A filament bundle is a cluster in which the filaments are suitably well aligned. We have analyzed the average structure of the filament hydrogel in phase diagrams as a function of the filament volume fraction $\phi$, the crosslinker-filament ratio $n_{l f}$, and the adhesion strength $\epsilon$. The phase diagram region, in which more than $20 \%$ of the filaments are part of a bundle, is called the bundle region. The percolation 
threshold is determined by studying the cluster size distribution $n_{s}$ and the fraction of filaments in the largest cluster $a_{\max }$ : At the percolation threshold, the fraction $n_{s}$ of clusters containing $s$ filaments is of the form $n_{s} \propto s^{\tau}$ with the Fisher exponent $\tau$. For a sufficiently large system, one has $\left\langle a_{\max }\right\rangle=0.5$ at the percolation threshold. In Figure 6a, phase diagram of the system is shown as a function of the adhesion strength $\epsilon / T$ and the filament volume fraction $\phi$ for fixed crosslinker-filament ratio $n_{l r}=2$. A percolated network forms if the filament volume fraction and the adhesion strength are large enough. This means, that a percolated network can be created and destroyed, reversibly, by changing the hydrogel volume or the temperature. Furthermore, the system forms bundles for suitably low filament volume fractions. This can be explained as follows: The system favors crosslinkers that are bound to filaments on both ends. Parallel filaments can be interconnected by a large number of crosslinks forming a ladder-like structure. At low filament concentrations, only aligned groups of filaments make it possible that large numbers of crosslinkers can bind on both ends. At high filament concentrations, crosslinkers can bind on both ends, even if the filament network is disordered. The phase diagram shows that percolated network may form with and without pronounced bundling and bundling can form with and without the formation of a percolated network.

The network and bundle formation can also be induced by increasing the crosslink-filament ratio. Another interesting aspect is the dependence of the percolation threshold on the filament length [25]. Interestingly, we found that for a given crosslinker-filament ratio $n_{l r}=2$ and a fixed filament volume fraction, the binding strength $\epsilon_{t}$ at which percolation sets in, is rather independent of the filament length that has been varied in the range between $l_{F}=10 D$ and $l_{F}=25 D$. The results could be reproduced qualitatively with a simple analytic model. Note, that the independence of the length is given at a fixed ratio $n_{l r}$ of filament to crosslink number density. In practice, the ratio $r$ of crosslinkers to filament monomers can be more important. For suitably large filament lengths one has $n_{l r} \simeq \frac{3}{8} r \frac{\ell_{F}}{D}$ so that the percolation threshold is independent of the filament length if $r \ell_{F}$ is kept fixed.

Figure 6. Model network with stiff filaments (blue) and short crosslinkers (yellow) with adhesive ends (red), studied with Monte Carlo simulations. (a) Snapshot of a network structure. (b) Phase diagram as a function of the adhesion strength over temperature $\epsilon / T$ and the filament volume fraction $\phi$. Figures from (R. Chelakkot, R. Lipowsky and T. Gruhn, Soft Matter, 2009, 5, 1504)—Reproduced with permission of The Royal Society of Chemistry.

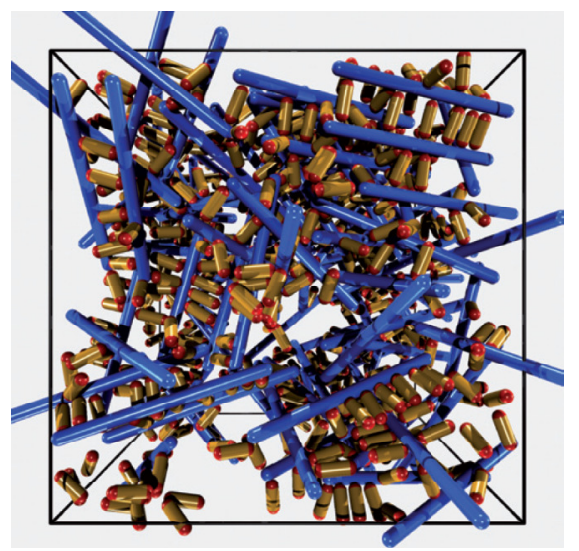

(a)

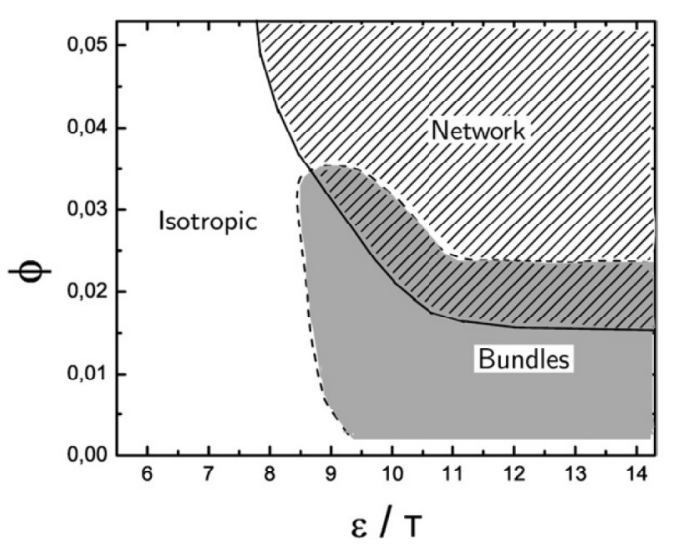

(b) 


\section{Summary and Conclusions}

Various macroscopic models and molecular simulation techniques have been developed, which allow the study of various kinds of stimuli-sensitive polymer networks. With these methods, the response of polymer networks on external stimuli can be analyzed and optimized on various time and length scales. The behavior of a hydrogel can depend, in a complex way, on many physical and chemical properties of the environment, such as the $\mathrm{pH}$ value, ion concentrations, the concentration of specific molecules, external electric fields, temperature, or illumination. The development and optimization of tailored, stimuli-sensitive polymer networks requires a good understanding of the interplay of all these aspects. Here, numerical modeling and simulations are crucial. Molecular simulations require a comparably low amount of parameters. For atomistic simulations only reasonable interatomic potentials are needed, which, in many cases, are provided by simulation software. However, time scales and system sizes that can be studied with atomistic simulations are small so that only a small number of network meshes can be studied. More coarse-grained simulations require model interaction parameters, which are often not available for the polymer network of interest. The parameters can be extracted from atomistic simulations on a small length scale or by comparing material properties of the model system with experimental measurements. If the parameters are found, very detailed information can be found about system properties like the swelling behavior of the network, the breaking and healing of bonds, changes of the polymer configurations and the network structure or, for networks with suitably small mesh sizes, the elastic and inelastic responses of the network on applied strains. Results of molecular simulations help to interpret the experimental measurements but they can also support macroscopic models, which depend on material properties such as the elastic constants of the polymer network for a given composition of the solvent or the permeability of a hydrogel for a specific molecule. For systems with chain lengths that are too large for molecular simulations, approaches like the self-consistent field theory can be a good alternative, especially, if the system consists of copolymers and forms nanostructures. Our extended self-consistent field theory allows the modeling of polymer systems on an intermediate length scale and helps to fill the gap between macroscopic theories and molecular simulations. Thus far, only a very small number of multi-scale methods have been used for the modeling of stimuli-sensitive networks on various length scales. This is one of the major tasks for the future.

One particularly important aspect of smart polymer networks is the response time. Quantitative predictions of absolute time scales are a big challenge for numerical methods. Many macroscopic models are restricted to stationary or steady-state systems. Macroscopic numerical studies of network dynamics are based on parameters like diffusion constants that have to be taken from molecular simulations or experiments. This shows, once more, the necessity of numerical studies on various levels of detail and of multiscale studies that combine these methods.

Another challenge is the numerical study of polymer networks with spatial inhomogeneities on the microscale rather than on the nanometer scale. Experimentally, such inhomogeneities can be inferred, for example, by differential photo-crosslinking of polymers [110] or by applying an irradiation pattern on a thermosensitive gel [111]. 
As documented in this article, numerical methods for small and large time scales are available such that in the future multi-scale simulations should play an important role for a comprehensive understanding of these fascinating and multifunctional materials.

\section{Acknowledgments}

The authors like to thank Daming Li for his contributions to the self-consistent field theory of reversibly crosslinked polymer networks and the phase field model of hydrogels. We also like to thank Hong Liu Yang for supporting the phase field studies of hydrogels. The simulations of the systems with stiff filament and crosslinkers have been carried out together with Raghunath Chelakkot and Reinhard Lipowsky, whom we would also like to thank. The work on this article was supported by DFG SPP 1259: "Intelligente Hydrogele, modeling and simulation of hydrogel swelling under strong non-equilibrium conditions using the phase-field and phase-field crystal methods" and the DFG SFB 840 "Von partikulären Nanosystemen zur Mesotechnologie".

\section{Conflict of Interest}

The authors declare no conflict of interest.

\section{References}

1. Jagur-Grodzinski, J. Polymeric gels and hydrogels for biomedical and pharmaceutical applications. Polym. Adv. Technol. 2010, 21, 27-47.

2. Adelsberger, J.; Metwalli, E.; Diethert, A.; Grillo, I.; Bivigou-Koumba, A.M.; Laschewsky, A.; Müller-Buschbaum, P.; Papadakis, C.M. Kinetics of collapse transition and cluster formation in a thermoresponsive micellar solution of P(S-b-NIPAM-b-S) induced by a temperature jump. Macromol. Rapid Commun. 2012, 33, 254-259.

3. Huck, W.T.S. Materials chemistry: Polymer networks take a bow. Nature 2011, 472, 425-426.

4. Suzuki, A.; Ishii, T.; Maruyama, Y. Optical switching in polymer gels. J. Appl. Phys. 1996, 80, 131-136.

5. Deligkaris, K.; Tadele, T.S.; Olthuis, W.; van den Berg, A. Hydrogel-based devices for biomedical applications. Sensor. Actuat. B-Chem. 2010, 147, 765-774.

6. Na, K.; Lee, E.S.; Bae, Y.H. Self-organized nanogels responding to tumor extracellular pH: pHdependent drug release and in vitro cytotoxicity against MCF-7 cells. Bioconjugate Chem. 2007, $18,1568-1574$.

7. Bassik, N.; Abebe, B.T.; Laflin, K.E.; Gracias, D.H. Photolithographically patterned smart hydrogel based bilayer actuators. Polymer 2010, 51, 6093-6098.

8. Holtz, J.H.; Asher, S.A. Polymerized colloidal crystal hydrogel films as intelligent chemical sensing materials. Nature 1997, 389, 829-832.

9. Nagai, D.; Suzuki, A.; Maki, Y.; Takeno, H. Reversible chain association/dissociation via a $\mathrm{CO}_{2}$ responsive crosslinking/decrosslinking system. Chem. Commun. 2011, 47, 8856-8858.

10. Çaykara, T.; Doğmuş, M. The effect of solvent composition on swelling and shrinking properties of poly(acrylamide-co-itaconic acid) hydrogels. Eur. Polym. J. 2004, 40, 2605-2609. 
11. Casolaro, M.; Casolaro, I. Multiple stimuli-responsive hydrogels for metal-based drug therapy. Polymers 2012, 4, 964-985.

12. Tan, L.; Liu, Y.; Ha, W.; Ding, L.-S.; Peng, S.-L.; Zhang, S.; Li, B.-J. Stimuli-induced gel-sol transition of multi-sensitive supramolecular $\beta$-cyclodextrin grafted alginate/ferrocene modified pluronic hydrogel. Soft Matter 2012, 8, 5746-5749.

13. Guenther, M.; Kuckling, D.; Corten, C.; Gerlach, G.; Sorber, J.; Suchaneck,G.; Arndt, K.-F. Chemical sensors based on multiresponsive block copolymer hydrogels. Sens. Actuators B 2007, 126, 97-106.

14. Seliktar, D. Designing cell-compatible hydrogels for biomedical applications. Science 2012, 336, 1124-1128.

15. Richter, A.; Howitz, S.; Kuckling, D.; Arndt, K.F. Influence of volume phase transition phenomena on the behavior of hydrogel-based valves. Sens. Actuators B: Chem. 2004, 99, 451-458.

16. Serra, L.; Doménech, J.; Peppas,N.A. Drug transport mechanisms and release kinetics from molecularly designed poly(acrylic acid-g-ethylene glycol) hydrogels. Biomaterials 2006, 27, 5440-5451.

17. Wu, C.; Ying, A.; Ren, S. Fabrication of polymeric micelles with core-shell-corona structure for applications in controlled drug release. Colloid. Polym. Sci. 2013, 291, 827-834.

18. Yue, J.; Wang, R.; Liu, S.; Wu, S.; Xie, Z.; Huang, Y.; Jing, X. Reduction-responsive shell-crosslinked micelles prepared from Y-shaped amphiphilic block copolymers as a drug carrier. Soft Matter 2012, 8, 7426-7435.

19. Yan, X.; Weng, F.; Zheng, B.; Huang, F. Stimuli-responsive supramolecular polymeric materials. Chem. Soc. Rev. 2012, 41, 6042-6065.

20. Li, D., Yang, H.L., Emmerich, H. Phase field model simulations of hydrogel dynamics under chemical stimulation. Colloid Polym. Sci. 2011, 289, 513-521.

21. Li, D.; Gruhn, T.; Emmerich, H. Mean field theory for a reversibly crosslinked polymer network. J. Chem. Phys. 2012, 137, 024906.

22. Gruhn, T.; Li, D.; Emmerich, H. Calculating structural properties of reversibly crosslinked polymer systems using self-consistent field theory. Prog. Colloid Polym. Sci. 2013, in press.

23. Gruhn, T.; Emmerich, H. Phase behavior of polymer blends with reversible crosslinks-A self-consistent field theory study. J. Mater. Res. 2013, doi:10.1557/jmr.2013.315.

24. Chelakkot, R.; Lipowsky, R.; Gruhn, T. Self-assembling network and bundle structures in systems of rods and crosslinkers - A Monte Carlo study. Soft Matter 2009, 5, 1504-1513.

25. Chelakkot, R.; Gruhn, T. Length dependence of crosslinker induced network formation of rods: a Monte Carlo study. Soft Matter 2012, 8, 11746-11754.

26. Saunders, J.R.; Abu-Salih, S.; Khaleque, T; Hanula, S; Moussa, W. Modeling theories of intelligent hydrogel polymers. J. Comput. Theor. Nanosci. 2008, 5, 1942-1960.

27. Shahinpoor, M.; Kim, K.J. Ionic polymer-metal composites-III. Modeling and simulation as biomimetic sensors, actuators, transducers and artificial muscles. Smart Materials and Structures 2004, 13, 1362-1388.

28. Wu, S.; Li, H.; Chen, J.P.; Lam, K.Y. Modeling investigation of hydrogel volume transition. Macromol. Theory Simul. 2004, 13, 13-29. 
29. Flory, P.J.; Rehner, J. Statistical mechanics of cross-linked polymer networks I. Rubberlike elasticity. J. Chem. Phys. 1943, 11, 512-520.

30. Flory, P.J.; Rehner, J. Statistical mechanics of cross-linked polymer networks II. Swelling. J. Chem. Phys. 1943, 11, 521-526.

31. Doi, M.; Matsumoto, M.; Hirose, Y. Deformation of ionic polymer gels by electric fields. Macromolecules 1992, 25, 5504-5511.

32. Erman, B.; Flory, P.J. Critical phenomena and transitions in swollen polymer networks and in linear macromolecules. Macromolecules 1986, 19, 2342-2353.

33. English, A.E.; Mafé, S.; Manzanares, J.; Yu, X.; Grosberg, A.Y. Equilibrium swelling properties of polyampholytic hydrogels. J. Chem. Phys. 1996, 104, 8713-8720.

34. Okay, O.; Sariisik, S.B. Swelling behavior of poly(acrylamide-co-sodium acrylate) hydrogels in aqueous salt solutions: Theory versus experiments. Eur. Polym. J. 2000, 36, 393-399.

35. Çaykara, T.; Bozkaya, U.; Kantoğlu Ö. Network Structure and Swelling Behavior of Poly(acrylamide/crotonic acid) Hydrogels in Aqueous Salt Solutions. J. Polym. Sci. Part B 2003, $41,1656-1664$.

36. Kramarenko, E.Y.; Khokhlov, A.R.; Yoshikawa, K. A three-state model for counterions in a dilute solution of weakly charged polyelectrolytes. Macromol. Theory Simul. 2000, 9, 249-256.

37. Keener, J.P.; Sircar, S.; Fogelson, A.L. Kinetics of swelling gels. Siam. J. Appl. Math. 2011, 71, 854-875.

38. Feng, L.; Jia, Y.; Chen, X.; Li, X.; An, L. A multiphasic model for the volume change of polyelectrolyte hydrogels. J. Chem. Phys. 2010, 133, 114904.

39. Chen, J.; Ma, G. Modelling deformation behaviour of polyelectrolyte gels under chemo-electromechanical coupling effects. Int. J. Numer. Meth. Engng. 2006, 68, 1052-1071.

40. Hon, Y.C.; Lu, M.W.; Xue, W.M.; Zhou, X. Numerical algorithm for triphasic model of charged and hydrated soft tissues. Comput. Mech. 2002, 29, 1-15.

41. Sun, D.N.; Gu, W.Y.; Guo, X.E.; Lai, W.M.; Mow, V.C. A mixed finite element formulation of triphasic mechano-electrochemical theory for charged, hydrated biological soft tissues. Int. J. Numer. Meth. Engng. 1999, 45, 1375-1402.

42. Freiboth, S.; Class, H.; Helmig, R.; Graf, T.; Ehlers, W.; Schwarz, W.; Vrettos, C. A model for multiphase flow and transport in porous media including a phenomenological approach to account for deformation-A model concept and its validation within a code intercomparison study. Comput. Geosci. 2009, 13, 281-300.

43. Zhou, X.; Hon, Y.C.; Sun, S.; Mak, A.F.T. Numerical simulation of the steady-state deformation of a smart hydrogel under an external electric field. Smart Mater. Struct. 2002, 11, 459-467.

44. De, S.K.; Aluru, N.R. A chemo-electro-mechanical mathematical model for simulation of $\mathrm{pH}$ sensitive hydrogels. Mech. Mater. 2004, 36, 395-410.

45. Hon, Y.C.; Lu, M.W.; Xue, W.M.; Zhou, X. A new formulation and computation of the triphasic model for mechano-electrochemical mixtures. Comput. Mech. 1999, 24, 155-165.

46. Chen, J.; Chen, X.; Hisada, T. Non-linear finite element analysis of mechanical electrochemical phenomena in hydrated soft tissues based on triphasic theory. Int. J. Numer. Meth. Engng. 2006, $65,147-173$. 
47. Wallmersperger, T.; Ballhause, D.; Kröplin, B.; Günther, M.; Gerlach, G. Coupled multi-field formulation in space and time for the simulation of intelligent hydrogels. J. Intel. Mat. Syst. Str. 2009, 20, 1483-1492.

48. Li, H.; Luo, R.; Lam, K.Y. Multiphysics modeling of electrochemomechanically smart microgels responsive to coupled pH/electric stimuli. Macromol. Biosci. 2009, 9, 287-297.

49. Li, H.; Luo, R. Modeling and characterization of glucose-sensitive hydrogel: Effect of Young's modulus. Biosens. Bioelectron. 2009, 24, 3630-3636.

50. Li, H.; Wang, X.; Wang, Z.; Lam, K.Y. Multiphysics modelling of volume phase transition of ionic hydrogels responsive to thermal stimulus. Macromol. Biosci. 2005, 5, 904-914.

51. Sun, S.; Mak, A.F.T. The dynamical response of a hydrogel fiber to electrochemical stimulation. J. Polym. Sci. Part B 2001, 39, 236-246.

52. Luo, R.; Li, H.; Lam, K.Y. Modeling and simulation of chemo-electro-mechanical behavior of pH-electric-sensitive hydrogel. Anal. Bioanal. Chem. 2007, 389, 863-873.

53. Wang, Q.X.; Li, H.; Lam, K.Y. Meshless Simulation of Equilibrium Swelling/Deswelling of PH-Sensitive Hydrogels. J. Polym. Sci. Part B 2006, 44, 326-337.

54. Li, H.; Ng, Y.; Cheng, J.Q.; Lam, K.Y. Hermite-Cloud: A novel true meshless method. Comput. Mech. 2003, 33, 30-41.

55. Li, H.; Mulay, S.S. 2D simulation of the deformation of $\mathrm{pH}$-sensitive hydrogel by novel strong-form meshless random differential quadrature method. Comput. Mech. 2011, 48, 729-753.

56. Li, H.; Ng, T.Y.; Yew, Y.K.; Lam, K.Y. Modeling and simulation of the swelling behavior of ph-stimulus-responsive hydrogels. Biomacromolecules 2005, 6, 109-120.

57. Anders, D.; Weinberg, K. A variational approach to the decomposition of unstable viscous fluids and its consistent numerical approximation. ZAMM Z. Angew. Math. Mech. 2011, 91, 609-629.

58. Tian, H.; Shao, J.; Ding, Y.; Li, Y.; Li, X. Numerical studies of electrically induced pattern formation by coupling liquid dielectrophoresis and two-phase flow. Electrophoresis 2011, 32, 2245-2252.

59. Boffetta, G.; Mazzino, A.; Musacchio, S.; Vozella, L. Rayleigh-Taylor instability in a viscoelastic binary fluid. J. Fluid mech. 2010, 643, 127-136.

60. $\mathrm{Xu}, \mathrm{H}$; Bellehumeur, C.T. Modeling the morphology development of ethylene copolymers in rotational molding. J. Appl. Polym. Sci. 2006, 102, 5903-5917.

61. Yue, P.; Feng, J.; Liu, C.; Shen, J. Diffuse-interface simulations of drop coalescence and retraction in viscoelastic fluids. J. Non-Newton. Fluid Mech. 2005, 129 163-176.

62. Emmerich, H. Advances of and by phase-field modelling in condensed-matter physics. Adv. Phys. 2008, 57, 1-87.

63. Cahn, J.; Hilliard, J. Free energy of a nonuniform system .1. Interfacial free energy. J. Chem. Phys. 1958, 28, 258-267.

64. Flory, P. Thermodynamics of high polymer solutions. J. Chem. Phys. 1942, 10, 51-61.

65. Huggins, M. Theory of solutions of high polymers. J. Am. Chem. Soc. 1942, 64, 1712-1719.

66. Li, Y.C.; Shi, R.P.; Wang, C.P., Liu, X.J.; Wang, Y. Predicting microstructures in polymer blends under two-step quench in two-dimensional space. Phys. Rev. E 2011, 83, 041502.

67. Bird, R.; Curtiss, C.; Hassager, O.; Armstrong, R. Dynamics of Polymeric Liquids; Volume 2, Wiley: New York, NY, USA, 1987. 
68. Oldroyd, J. The effect of interfacial stabilizing films on the elastic and viscous properties of emulsions. Proc. R. Soc. A 1955, 232, 567-577.

69. Beaucourt, J.; Biben, T.; Verdier, C. Elongation and burst of axisymmetric viscoelastic droplets: a numerical study. Phys. Rev. 2005, E71, 066309.

70. Yue, P.; Zhou, C.; Feng, J.J. A computational study of the coalescence between a drop and an interface in newtonian and viscoelastic fluids. Phys. Fluids 2006, 18, 102102.

71. Hohenberg, P.; Halperin, B. Theory of dynamic critical phenomena. Rev. Mod. Phys. 1977, 49, 435-479.

72. Badalassi, V.; Ceniceros, H.; Banerjee, S. Computation of multiphase systems with phase field models. J. Comput. Phys. 2003, 190, 371-397.

73. Müller-Krumbhaar,H.; Emmerich, H.; Brener, E.; Hartmann, M. Dewetting hydrodynamics in 1+1 dimensions. Phys. Rev. E 2001, 63, 026304.

74. Allen, S.; Cahn, J. Microscopic theory for antiphase boundary motion and its application to antiphase domain coarsening. Acta metall. 1979, 27, 1085-1095.

75. Wang, D.; Shi, T.; Chen, J.; An, L.; Jia, Y. Simulated morphological landscape of polymer single crystals by phase field model. J. Chem. Phys. 2008, 129, 194903.

76. $\mathrm{Xu}, \mathrm{H}$.; Matkar, R.; Kyu, T. Phase-field modeling on morphological landscape of isotactic polystyrene single crystals. Phys. Rev. E 2005, 72, 011804.

77. Ricka, J.; Tanaka, T. Swelling of ionic gels: Quantitative performance of the Donnan theory. Macromolecules 1984, 17, 2916-2921.

78. Edwards, S.F. The statistical mechanics of polymers with excluded volume. Proc. Phys. Soc. 1965, 85, 613-624.

79. Helfand, E.; Yukiko, T. Theory of the interface between immiscible polymers. II. J. Chem. Phys. 1972, 56, 3592-3601.

80. Binder, K.; Milchev, A. Polymer brushes on flat and curved surfaces: How computer simulations can help to test theories and to interpret experiments. J. Polym. Sci., Part B: Polym. Phys. 2012 $50,1515-1555$.

81. Wang, J.; Müller, M.; Wang, Z.-G. Nucleation in A/B/AB blends: Interplay between microphase assembly and macrophase separation. J. Chem. Phys. 2009, 130, 154902.

82. Müller, M.; Schmid, F. Incorporating fluctuations and dynamics in self-consistent field theories for polymer blends. Adv. Polym. Sci. 2005, 185, 1-56.

83. Fredrickson, G.H.; Ganesan, V.; Drolet, F. Field-theoretic computer simulation methods for polymers and complex fluids. Macromolecules 2002, 35, 16-39.

84. Schmid, F. Self-consistent-field theories for complex fluids. J. Phys.: Condens. Matter 1998, 10, 8105-8138.

85. Benhamou, M.; Derouiche, A.; Bettachy, A.; F. Elhajjaji, F. Critical microphase properties of crosslinked polymer blends with quenched random impurities. Eur. Phys. J. E 2011, 34, 1-8.

86. Mao, X.; Goldbart, P.M.; Xing, X.; Zippelius, A. Soft random solids and their heterogeneous elasticity. Phys. Rev. E 2009, 80, 031140.

87. Ulrich, S.; Mao, X.; Goldbart, M.; Zippelius, A. Elasticity of highly cross-linked random networks. Europhys. Lett. 2006, 76, 677-682. 
88. Helfand, E. Theory of inhomogeneous polymers: Fundamentals of the Gaussian random-walk model. J. Chem. Phys. 1975, 62, 999-1005.

89. Düchs, D.; Ganesan, V.; Fredrickson, G.H.; Schmid, F. Fluctuation effects in ternary AB+A+B polymeric emulsions. Macromolecules 2003, 36, 9237-9248.

90. Bates, F.S.; Maurer, W.W.; Lipic, P.M.; Hillmyer, M.A.; Almdal, K.; Mortensen, K.; Fredrickson, G.H.; Lodge, T.P. Polymeric bicontinuous microemulsions. Phys. Rev. Lett. 1997, 79, 849-852.

91. Hillmyer, M.A.; Maurer, W.W.; Lodge, T.P.; Bates, F.S.; Almdal, K.J. Model bicontinuous microemulsions in ternary homopolymer/block copolymer blends. Phys. Chem. 1999, 103, 4814-4824.

92. Secchi, E.; Roversi, T.; Buzzaccaro, S.; Piazza, L.; Piazza, R. Biopolymer gels with "physical" cross-links: gelation kinetics, aging, heterogeneous dynamics, and macroscopic mechanical properties. Soft Matter 2013, 9, 3931-3944.

93. Allen, M.P.; Tildesley, D.J. Computer Simulations of Liquids, Reprint ed.; Oxford University Press: Oxford, Great Britain, 1993.

94. Frenkel, D.; Smith, B. Understanding Molecular Simulation, 2nd ed.; Academic Press: San Diego, CA, USA, 2002.

95. Kotelyanskii, M.; Theodorou, D.N. Simulation Methods for Polymers, 1st ed.; Marcel Dekker Inc.: New York, NY, USA, 2004.

96. Masoud, H.; Alexeev, A. Controlled release of nanoparticles and macromolecules from responsive microgel capsules. ACS Nano 2012, 6, 212-219.

97. Netz, R.R. Polyelectrolytes in electric fields. J. Phys. Chem. B 2003, 107, 8208-8217.

98. Phadke, A.; Zhang, C.; Arman, B.; Hsu, C.-C.; Mashelkar, R.A.; Lele, A.K.; Tauber, M.J.; Arya, G.; Varghese, S. Rapid self-healing hydrogels. Proc. Natl. Acad. Sci. USA 2012, 109, 4383-4388.

99. Trewin, A.; Willock, D.J.; Cooper, A.I. Atomistic simulation of micropore structure, surface area, and gas sorption properties for amorphous microporous polymer networks. J. Phys. Chem. C 2008, 112, 20549-20559.

100. Jang, S.S.; Goddard, W.A., 3rd; Kalani, M.Y.S. Mechanical and transport properties of the poly(ethylene oxide)-poly(acrylic acid) double network hydrogel from molecular dynamic simulations. J. Phys. Chem. B 2007, 111, 1729-1737.

101. Wu, C.; Xu, W. Atomistic simulation study of absorbed water influence on structure and properties of crosslinked epoxy resin. Polymer 2007, 48, 5440-5448.

102. Liu, H.; Li, M.; Lu, Z.-Y.; Zhang, Z.G.; Sun, C.-C.; Cui, T. Multiscale simulation study on the curing reaction and the network structure in a typical epoxy system. Macromolecules 2011, 44, $8650-8660$.

103. Deshmukh, S.; Mooney, D.A.; McDermott, T.; Kulkarnib, S.; Don MacElroya, J.M. Molecular modeling of thermo-responsive hydrogels: observation of lower critical solution temperature. Soft Matter 2009, 5, 1514-1521.

104. Aydt, E.M.; Hentschke, R. Swelling of a model network: A Gibbs-ensemble molecular dynamics study. J. Chem. Phys. 2000, 112, 5480-5487. 
105. Quesada-Pérez, M.; Maroto-Centeno, J.A.; Martín-Molina, A. Effect of the counterion valence on the behavior of thermo-sensitive gels and microgels: A Monte Carlo Simulation Study. Macromolecules 2012, 45, 8872-8879.

106. Schneider, S.; Linse, P. Swelling of cross-linked polyelectrolyte gels. Eur. Phys. J. E 2002, 8, 457-460.

107. Schneider, S.; Linse, P. Discontinuous volume transitions in cross-linked polyelectrolyte gels induced by short-range attractions and strong electrostatic coupling. Macromolecules 2004, 37, 3850-3856.

108. Guo, L.; Luijten, E.; Reversible gel formation of triblock copolymers studied by molecular dynamics simulation. J. Polym. Sci. Part B 2005, 43, 959-969.

109. Schneider, S.; Linse, P. Monte carlo simulation of defect-free cross-linked polyelectrolyte gels. $J$. Phys. Chem. B 2003, 107, 8030-8040.

110. Jamal, M.; Zarafshar, A.M.; Gracias, D.H. Differentially photo-crosslinked polymers enable self-assembling microfluids. Nature Commun. 2011, 2, 527.

111. Kim, J.; Hanna, J.A.; Byun, M.; Santangelo C.D.; Hayward, R.C. Designing Responsive Backled Surfaces by Halftone Gel Lithography. Science 2012, 335, 1201-1205.

(C) 2013 by the authors; licensee MDPI, Basel, Switzerland. This article is an open access article distributed under the terms and conditions of the Creative Commons Attribution license (http://creativecommons.org/licenses/by/3.0/). 